\title{
Epidemiologische Untersuchung zum Sterbeort von Tumorpatienten
}

\section{Auswertung von Todesbescheinigungen einer Dekade}

\author{
B. Dasch ${ }^{1}$, K. Blum², H. Vogelsang ${ }^{3}$, C. Bausewein ${ }^{1}$
}

Institute

1 Klinik und Poliklinik für

Palliativmedizin, Universität München

2 Praxisgemeinschaft Gartenstraße, Bochum

3 Klinik für Anästhesiologie und operative Intensivmedizin St. Josef-Hospital, Universität Bochum

\section{Korrespondenz}

Dr. med. Burkhard Dasch Klinik und Poliklinik für Palliativmedizin Klinikum der Universität München

Marchioninistr. 15 81377 München

Burkhard.Dasch@med. uni-muenchen.de

\section{Zusammenfassung}

Hintergrund I In Deutschland wird der Sterbeort aus Todesbescheinigungen nicht evaluiert. Deshalb gibt es auch für Tumorpatienten kaum populationsbezogene Sterbeortdaten. Ziel der Untersuchung war es, die Veränderung des Sterbeortes von Tumorpatienten über einen Zeitraum von 10 Jahren zu beschreiben.

Material und Methodik | Todesbescheinigungen ausgewählter Regionen in Westfalen-Lippe der Jahre 2001 und 2011 wurden ausgewertet. Anhand ärztlicher Angaben zur Todesursache wurden Tumorpatienten identifiziert. Die Häufigkeitsverteilung des Sterbeortes wurde deskriptiv dargestellt. Zudem wurden Subgruppenanalysen nach Tumorentität (ICD-10, C00-C96) durchgeführt.

Ergebnisse I Analysiert wurden 24009 Todesbescheinigungen (2001: 11585; 2011: 12424). Bei $34,0 \%$ war eine Tumorerkrankung beschrieben. Folgende Sterbeortverteilung lag vor (2001 vs.
2011): häusliches Umfeld $24,1 \%$ vs. $24,7 \%$ ( $p=0,553)$, Krankenhaus $62,8 \%$ vs. $51,4 \%$ $(\mathrm{p}=0,001)$, Palliativstation $0,0 \%$ vs. $2,2 \%$, Altenoder Pflegeheim $7,4 \%$ vs. $10,9 \%(p=0,001)$, Hospiz $5,5 \%$ vs. $12,5 \%(p=0,001)$, sonstiger Ort $0,1 \%$ vs. $0,3 \%(p=0,063)$, keine Angabe $0,1 \%$ vs. $0,3 \%$ $(p=0,015)$. Patienten mit einer bösartigen Neubildung des ZNS (C69-C72) starben überproportional häufig im Hospiz (2011: Frauen 23,5\%; Männer 27,7\%), Patienten mit einer bösartiger Neubildung des lymphatischen und hämatopoetischen Gewebes (C81-C96) besonders häufig im Krankenhaus (2011: Frauen 63,7\%; Männer $68,4 \%)$.

Folgerung | Tumorpatienten sterben überwiegend in Institutionen, wobei das Krankenhaus den häufigsten Sterbeort darstellt. Nur jeder vierte Sterbefall findet im häuslichen Umfeld statt. Tendenziell kann eine Verlagerung des Sterbeortes weg vom Krankenhaus, hin besonders zu Hospizen und Palliativstationen aber auch zu Altenheimen beobachtet werden.

\section{Einleitung}

Der Sterbeort wird in der ärztlichen Todesbescheinigung zwar dokumentiert, jedoch nicht weiter statistisch ausgewertet. Dementsprechend liegen auch für Tumorpatienten kaum bevölkerungsbezogene Sterbeortdaten vor.

In Deutschland starben im Jahre 2014 laut Angaben des Statistischen Bundesamtes 223758 Personen an einer bösartigen Tumorerkrankung (ICD-10, C00-C97) [1]. Dies entspricht einem prozentualen Anteil von 25,8\% aller Sterbefälle. Tumorerkrankungen rangieren hinter den HerzKreislauf-Erkrankungen an zweiter Stelle aller Todesursachen.

Befragungen zeigen, dass Patienten mit einer fortgeschrittenen Tumorerkrankung sich mehrheitlich für ein Sterben im häuslichen Umfeld aussprechen, jedoch dieser Wunsch in der Realität oft nicht erfüllt werden kann [2-5]. Das Sterben findet vorwiegend in Institutionen statt, wobei das Krankenhaus hier den häufigsten Sterbeort darstellt [6-15].
In den zurückliegenden zwei Jahrzehnten hat die Betreuung Schwerstkranker und Sterbender in ihrer letzten Lebensphase stark an gesellschaftlicher Aufmerksamkeit gewonnen. Die Schärfung dieses Bewusstseins wurde nicht zuletzt durch das Anliegen und die Tätigkeit der Hospizbewegung und der Palliativmedizin, sich für ein menschenwürdiges Sterben einzusetzen, vorangetragen. Mit dem Gesetz zum Ausbau der Hospiz- und Palliativversorgung wird nun auf politischer Ebene der Versuch unternommen, diesem gesellschaftlichen Anliegen Rechnung zu tragen [16].

Patienten mit einem fortgeschrittenen Tumorleiden stellen die weitaus größte Patientengruppe hospizlich und palliativmedizinisch betreuter Personen dar. Es ist aber wenig über die Sterbeorte von Tumorpatienten bekannt und wie sich diese im Verlauf der Zeit ändern. Das Studienziel war es daher, den Sterbeort von Tumorpatienten über eine Dekade zu beschreiben. Zudem sollten Subgruppenanalysen zeigen, welche Zusammen- 


\begin{tabular}{|c|c|c|c|c|c|c|}
\hline \multirow[b]{2}{*}{ Jahr } & \multicolumn{2}{|c|}{ Gesamt } & \multicolumn{2}{|c|}{ Stadt } & \multicolumn{2}{|c|}{ Land } \\
\hline & 2001 & 2011 & 2001 & 2011 & 2001 & 2011 \\
\hline Bevölkerung (n) & 1231222 & 1243957 & 656756 & 654540 & 574466 & 578024 \\
\hline Bevölkerungsdichte (pro km²) & 414 & 418 & 1466 & 1461 & 227 & 229 \\
\hline Krankenhausbetten (pro 1000) & 8,1 & 7,8 & 10,6 & 10,6 & 5,2 & 4,8 \\
\hline Pflegeheimbetten (pro 1000) & 7,6 & 8,9 & 8,1 & 9,6 & 7,1 & 8,3 \\
\hline Hospize (n) & 3 & 6 & 3 & 3 & 0 & 3 \\
\hline Hospizbetten (n) & 27 & 50 & 27 & 31 & 0 & 19 \\
\hline Hospizbetten (pro 100 000) & 2,2 & 4,0 & 4,1 & 4,7 & 0 & 3,3 \\
\hline Palliativstationen (n) & 0 & 6 & 0 & 3 & 0 & 3 \\
\hline
\end{tabular}

hänge es zwischen Tumorentität (ICD-10, C00C96) und Sterbeort gibt.

\section{Methoden}

Studiendesign I Retrospektive statistische Auswertung von Totenscheinen der Jahre 2001 und 2011 nach dem Sterbeort. Die vorliegende Analyse stellt hier die Auswertungsergebnisse für Tumorpatienten dar.

Studienregion | Die Studienregion umfasste Teile von Westfalen-Lippe, wobei die Universitätsstädte Münster (MS) und Bochum (BO) sowie die Landkreise Borken (BOR) und Coesfeld (COE) ausgewählt wurden. Die Auswahl der Regionen erfolgte zufällig, jedoch wurde darauf geachtet, dass sowohl städtische wie ländliche Regionen Westfalens ausgewählt wurden, um Vergleichsanalysen durchführen zu können. Zum Stichtag 31.12.2010 lebten in der Studienregion 1243957 Personen. Zwischen 2001 und 2011 erhöhte sich die Einwohnerzahl um 1,03\% [17].

Im Jahre 2001 existierten in den Städten drei Hospize (Bo 1, MS 2) mit einer Bettenzahl von 4,1 pro 100000 Einwohner. Die Bettenzahl blieb in der Stadt über zehn Jahre annähernd konstant. In den Landkreisen gab es im Jahr 2001 kein Hospiz, jedoch wurden hier im weiteren Verlauf drei neue Hospize errichtet (BOR 2, COE 1). Palliativstationen wurden erst nach 2001 gegründet (BO 1, MS 2, BOR 2, COE 1) ( Tab. 1)

Definition des Sterbeortes | Der Sterbeort wurde unterteilt in die Kategorien

- häusliches Umfeld,

- Krankenhaus,

- Palliativstation,

- Alten- oder Pflegeheim,

- Hospiz und

- sonstiger Ort.

Unter der Kategorie häusliches Umfeld wurde die Privatwohnung des Verstorbenen oder die Wohnung von Familienangehörigen zusammenge- fasst. Krankenhäuser, psychiatrische Kliniken und Kurkliniken wurden zum Sterbeort Krankenhaus subsumiert. Palliativstationen zählten als eigenständiger Sterbeort. Die Kategorie Altenoder Pflegeheim umfasste alle Einrichtungen der Altenheime, Altenwohnheime, Altenpflegeheime, betreutes Wohnen sowie Kurzzeitpflegen. Sonstige Orte betrafen unter anderem öffentliche Plätze, Hausarztpraxen, Freizeiteinrichtungen.

Auswertung der Totenscheine | Die Studie konnte auf einen vollständigen Datensatz aller Todesbescheinigungen der Jahre 2001 und 2011 zurückgreifen. Ausgewählt wurde ein zehnjähriger Beobachtungszeitraum, da zu Beginn der Beobachtung im Jahre 2001 kaum hospizliche und palliativmedizinische Versorgungsstrukturen vorhanden waren und diese erst aufgebaut werden mussten. Eine Vernichtung von Leichenschauscheinen aufgrund von Verjährungsfristen lag nicht vor. In der vorliegenden Auswertung wurden alle Todesfälle einbezogen, bei denen eine natürliche Todesursache dokumentiert war. Dokumente, bei denen keine Information zum Alter oder Geschlecht vorlagen, wurden von der Analyse ausgeschlossen. Insgesamt wurden 24877 Totenscheine ausgewertet (2001: 11963, 2011: 12914). Bei 126 Dokumenten fehlten Angaben zum Alter oder Ge-

\section{Rohe Mortalitätsrate von Tumorpatienten}

\begin{tabular}{|c|c|c|c|c|}
\hline Gesamt & $\begin{array}{l}\text { alle } \\
\text { (24009) }\end{array}$ & $\begin{array}{l}\text { Frauen } \\
(12651)\end{array}$ & $\begin{array}{l}\text { Männer } \\
(11358)\end{array}$ & p-Wert \\
\hline Tumorpatienten & $\begin{array}{l}34,0 \% \\
(8172)\end{array}$ & $\begin{array}{l}30,4 \% \\
(3844)\end{array}$ & $\begin{array}{l}38,1 \% \\
(4328)\end{array}$ & 0,001 \\
\hline 2001 & $\begin{array}{l}\text { alle } \\
\text { (11585) }\end{array}$ & $\begin{array}{l}\text { Frauen } \\
(6214)\end{array}$ & $\begin{array}{l}\text { Männer } \\
\text { (5371) }\end{array}$ & p-Wert \\
\hline Tumorpatienten & $\begin{array}{l}33,2 \% \\
(3844)\end{array}$ & $\begin{array}{l}29,9 \% \\
(1855)\end{array}$ & $\begin{array}{l}37,0 \% \\
(1989)\end{array}$ & 0,001 \\
\hline 2011 & $\begin{array}{l}\text { alle } \\
\text { (12424) }\end{array}$ & $\begin{array}{l}\text { Frauen } \\
(6437)\end{array}$ & $\begin{array}{l}\text { Männer } \\
\text { (5987) }\end{array}$ & p-Wert \\
\hline Tumorpatienten & $\begin{array}{l}34,8 \% \\
(4328)\end{array}$ & $\begin{array}{l}30,7 \% \\
(1973)\end{array}$ & $\begin{array}{l}39,3 \% \\
(2355)\end{array}$ & 0,001 \\
\hline
\end{tabular}

Tab. 1 Strukturdaten der Studienregion im zeitlichen Vergleich.

Tab. 2 Rohe Mortalitätsrate von Tumorpatienten.

(2355) 


\begin{tabular}{|c|c|c|c|c|}
\hline & Gesamt & 2001 & 2011 & p-Wert \\
\hline Gesamt & $n=8172$ & $n=3844$ & $n=4328$ & \\
\hline Frauen, \% (n) & $\begin{array}{l}46,8 \\
(3828)\end{array}$ & $\begin{array}{l}48,3 \\
(1855)\end{array}$ & $\begin{array}{l}45,6 \\
(1973)\end{array}$ & 0,016 \\
\hline Männer, \% (n) & $\begin{array}{l}53,2 \\
(4344)\end{array}$ & $\begin{array}{l}51,7 \\
(1989)\end{array}$ & $\begin{array}{l}54,4 \\
(2355)\end{array}$ & 0,016 \\
\hline Alter, MW (STD) & $72,6(13,4)$ & $71,7(13,6)$ & $73,3(13,2)$ & 0,059 \\
\hline Häusliches Umfeld & $n=1998$ & $n=928$ & $n=1070$ & \\
\hline Frauen, \% (n) & $\begin{array}{l}45,8 \\
(915)\end{array}$ & $\begin{array}{l}47,3 \\
(439)\end{array}$ & $\begin{array}{l}44,5 \\
(476)\end{array}$ & 0,208 \\
\hline Männer, \% (n) & $\begin{array}{l}54,2 \\
(1083)\end{array}$ & $\begin{array}{l}52,7 \\
(489)\end{array}$ & $\begin{array}{l}55,5 \\
(594)\end{array}$ & 0,208 \\
\hline Alter, MW (STD) & $73,7(13,2)$ & $73,9(13,3)$ & $73,6(13,2)$ & 0,881 \\
\hline Krankenhaus & $n=4638$ & $n=2414$ & $n=2224$ & \\
\hline Frauen, \% (n) & $\begin{array}{l}42,4 \\
(1965)\end{array}$ & $\begin{array}{l}44,5 \\
(1075)\end{array}$ & $\begin{array}{l}40,0 \\
(890)\end{array}$ & 0,002 \\
\hline Männer, \% (n) & $\begin{array}{l}57,6 \\
(2673)\end{array}$ & $\begin{array}{l}55,5 \\
(1339)\end{array}$ & $\begin{array}{l}60,0 \\
(1334)\end{array}$ & 0,002 \\
\hline Alter, MW (STD) & $70,5(13,3)$ & $69,6(13,4)$ & $71,6(13,2)$ & 0,331 \\
\hline Palliativstation & $n=96$ & $\mathrm{n}=\mathbf{0}$ & $n=96$ & \\
\hline Frauen, \% (n) & $56,3(54)^{\#}$ & - & $56,3(54)$ & $*$ \\
\hline Männer, \% (n) & $43,7(42)^{\#}$ & - & $43,7(42)$ & $*$ \\
\hline Alter, MW (STD) & $72,6(10,8)^{\#}$ & - & $72,6(10,8)$ & * \\
\hline Alten- od. Pflegeheim & $n=754$ & $n=284$ & $n=470$ & \\
\hline Frauen, \% (n) & $68,4(516)$ & $74,6(212)$ & $64,7(304)$ & 0,005 \\
\hline Männer, \% (n) & $31,6(238)$ & $25,4(72)$ & $35,3(166)$ & 0,005 \\
\hline Alter, MW (STD) & $83,0(9,7)$ & $82,3(10,3)$ & $83,4(9,3)$ & 0,023 \\
\hline Hospiz & $n=751$ & $\mathrm{n}=\mathbf{2 1 2}$ & $n=539$ & \\
\hline Frauen, \% (n) & $55,9(420)$ & $59,9(127)$ & $54,4(293)$ & 0,191 \\
\hline Männer, \% (n) & $44,1(331)$ & $40,1(85)$ & $45,6(246)$ & 0,191 \\
\hline Alter, MW (STD) & $71,7(12,6)$ & $71,9(13,0)$ & $71,7(12,5)$ & 0,440 \\
\hline
\end{tabular}

Tab. 3 Charakteristika verstorbener Tumorpatienten. MW: Mittelwert; STD: Standardabweichung

schlecht. Bei 742 Todesfällen ergaben sich Anhaltspunkte für das Vorliegen eines nichtnatürlichen Todes. Letztendlich wurden 24009 Todesbescheinigungen in die Auswertungen aufgenommen (2001: 11585, 2011: 12424).

Tumorpatienten | Personen mit einer bösartigen Tumorerkrankung wurden anhand schriftlicher Arztangaben zur Todesursache identifiziert. Hierbei wurden sämtlich verfügbaren Angaben zur Todesursache ausgewertet, einschließlich ärztlicher Epikrise. Anschließend erfolgte eine Einteilung der bösartigen Neubildungen (C00-C96) gemäß der internationalen statistischen
Klassifikation von Krankheiten (ICD-10), zuzüglich Unterteilung nach Geschlecht. Aufgrund niedriger Fallzahlen wurden Patienten mit einer bösartigen Neubildung des Knochens und Gelenkknorpels (C40-C41) sowie der Schilddrüse, der Nebenniere und sonstigen endokrinen Drüsen (C73-C75) zu einer gemeinsamen Gruppe zusammengefasst. Verstorbene Personen, bei denen zwar eine Tumorerkrankung beschrieben war, diese jedoch nicht ausreichend war, um eine Klassifikation durchführen zu können, wurden in die Patientengruppe „nicht klassifizierbar“ zusammengefasst. Diese Gruppe umfasste $n=349$ Personen, entsprechend einem Anteil von 4,3\% aller verstorbenen Krebspatienten. Im Vergleich zu Patienten, deren Tumorentität klassifiziert werden konnte, lag hier der Anteil älterer Personen über 80 Jahre leicht höher, was ebenso den etwas stärkeren Anteil Verstorbener im Altenoder Pflegeheim erklärt.

Ethikkommission I Die Studie wurde bei der Ethik-Kommission der Ruhr-Universität Bochum beantragt und genehmigt (Registrier-Nr. 452212). Die Gesundheitsämter wurden mit der Bitte angeschrieben, Einsicht in die dort archivierten Todesbescheinigungen zu gewähren. Unter Wahrung gesetzlich vorgeschriebener Datenschutzbedingungen wurde die Datenerhebung sowie wissenschaftliche Auswertung von amtlicher Seite genehmigt.

Statistische Auswertungen | Die Häufigkeit von verstorbenen Tumorpatienten (Gesamtjahre sowie Jahre 2001 und 2011) wurde auf Geschlechtsunterschiede untersucht. Zudem wurde die Studienpopulation hinsichtlich Geschlecht und Durchschnittsalter untergliedert nach Sterbeort deskriptiv beschrieben und auf Unterschiede im zeitlichen Verlauf (2001 vs. 2011) getestet. Ebenso wurde der Sterbeort von Tumorpatienten (häusliches Umfeld, Krankenhaus, Palliativstation, Altenod. Pflegeheim, Hospiz, sonstiger Ort, keine Angabe) auf Unterschiede in der zeitlichen Häufigkeitsverteilung (2001 vs. 2011) statistisch untersucht. Stratifiziert nach Tumorentität wurde die Verteilung des Sterbeortes aufgegliedert nach Geschlecht prozentual dargestellt.

Für stetige Daten wurde der unverbundenen tTests angewendet, bei kategoriellen Daten der Chi-Quadrat-Test, bzw. bei niedriger Häufigkeit der Zellzahl kleiner 5 der Fisher-exakt-Test. Hierbei besagt die Nullhypothese, dass die Werteausprägungen der untersuchten Variablen gemäß Geschlecht bzw. Untersuchungszeit unabhängig voneinander auftreten. Die Alternativhypothese hingegen zeigt an, dass die untersuchten Variablen aufgrund bestimmter Werteausprägungen miteinander assoziiert sind. Das Signifikanzniveau wurde bei $\mathrm{p}<0,05$ (zweiseitig) festgelegt. Die Auswertungen erfolgten mit SPSS-Version 21. 


\begin{tabular}{|lllll}
\hline & Gesamt & $\mathbf{2 0 0 1}$ & $\mathbf{2 0 1 1}$ & p-Wert \\
\hline & $\mathrm{n}=8172$ & $\mathrm{n}=3844$ & $\mathrm{n}=4328$ & 0,553 \\
\hline häusliches Umfeld, \% (n) & $24,4(1998)$ & $24,1(928)$ & $24,7(1070)$ & 0,001 \\
\hline Krankenhaus, \% (n) & $56,8(4638)$ & $62,8(2414)$ & $51,4(2224)$ & $*$ \\
\hline Palliativstation, \% (n) & $1,2(96)^{\#}$ & - & $2,2(96)$ & 0,001 \\
\hline Alten- od. Pflegeheim, \% (n) & $9,2(754)$ & $7,4(284)$ & $10,9(470)$ & 0,001 \\
\hline Hospiz, \% (n) & $9,2(751)$ & $5,5(212)$ & $12,5(539)$ & 0,063 \\
\hline sonstiger Ort, \% (n) & $0,2(14)$ & $0,1(3)$ & $0,3(11)$ & 0,015 \\
\hline keine Angabe, \% (n) & $0,2(17)$ & $0,1(3)$ & $0,3(14)$ &
\end{tabular}

\& inklusive Palliativstationen, " Daten von 2001, * 2001 gab es noch keine Palliativstationen in der Studienregion

\section{Ergebnisse}

In Durchschnitt war bei 34,0\% $(\mathrm{n}=8172)$ aller ausgestellten Todesbescheinigungen eine Tumorerkrankung dokumentiert. Hierbei stieg der Prozentsatz von 33,2\% (2001) auf 34,8\% (2011) an. Bei Männern lag der Anteil (2001 vs. 2011) bei $37,0 \%$ vs. $39,3 \%$, bei Frauen bei $29,9 \%$ vs. $30,7 \%$ ( Tab. 2).

Das durchschnittliche Sterbealter aller verstorbenen Tumorerkrankten betrug im Jahre 2001 71,7 Jahre und stieg innerhalb von 10 Jahren auf 73,3 Jahre an. Tumorpatienten, die im Krankenhaus starben, wiesen verglichen zu den übrigen Sterbeorten das niedrigste Durchschnittsalter auf. Im Geschlechtervergleich verstarben Frauen prozentual häufiger im Alten- oder Pflegeheim sowie Hospiz und Männer häufiger im häuslichen Umfeld und Krankenhaus ( $\bullet$ Tab. 3).

Über die Hälfte aller Tumorpatienten starb im Krankenhaus, knapp ein Viertel zu Hause und annähernd jeder Zehnte im Hospiz oder Alten- bzw.
Pflegeheim. Tendenziell ging der Anteil stationär Verstorbener um 11,4\% Prozent zurück. Andererseits stieg die Häufigkeit im Hospiz Verstorbener deutlich um mehr als das Doppelte an (5,5\% vs. $12,5 \%, p=0,001)$. Alten- oder Pflegeheime verzeichneten einen Zuwachs von 3,5\%. Die Häufigkeit häuslicher Sterbefälle blieb mit 24,1 \% (2001) bzw. 24,7\% (2011) annährend konstant ( Tab. 4).

Im Geschlechtervergleich ergab sich, dass männliche Tumorpatienten im Krankenhaus häufiger starben, andererseits Frauen den deutlich größeren Anteil verstorbener Tumorpatienten im Hospiz sowie Alten- oder Pflegeheim repräsentierten. Im zeitlichen Trend wiesen Männer wie Frauen eine ähnliche Sterbeortverteilung auf ( $\triangleright$ Abb. 1).

Stratifiziert nach Tumorentität (C00-C96) und Geschlecht starben Patienten mit einer bösartigen Neubildung des lymphatischen und hämatopoetischen Gewebes (C81-C96) besonders häufig im Krankenhaus (Frauen: 79,4\% bzw. 63,7\%; Männer: 77,5\% bzw. 68,4\%). Im Gegensatz dazu wiesen Patienten mit einer bösartigen Neubildung des ZNS
Tab. 4 Sterbeorte von Tumorpatienten.

Abb. 1 Zeitlicher Trend der Sterbeortverteilung im Geschlechtervergleich.

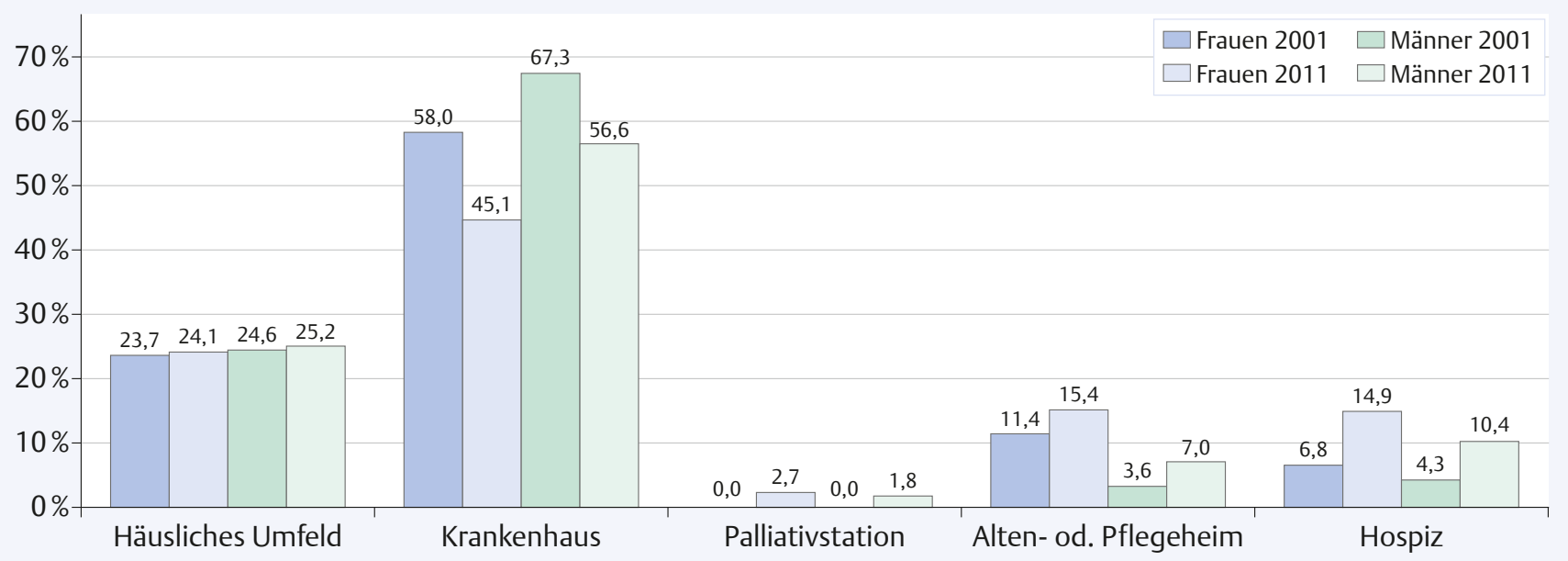




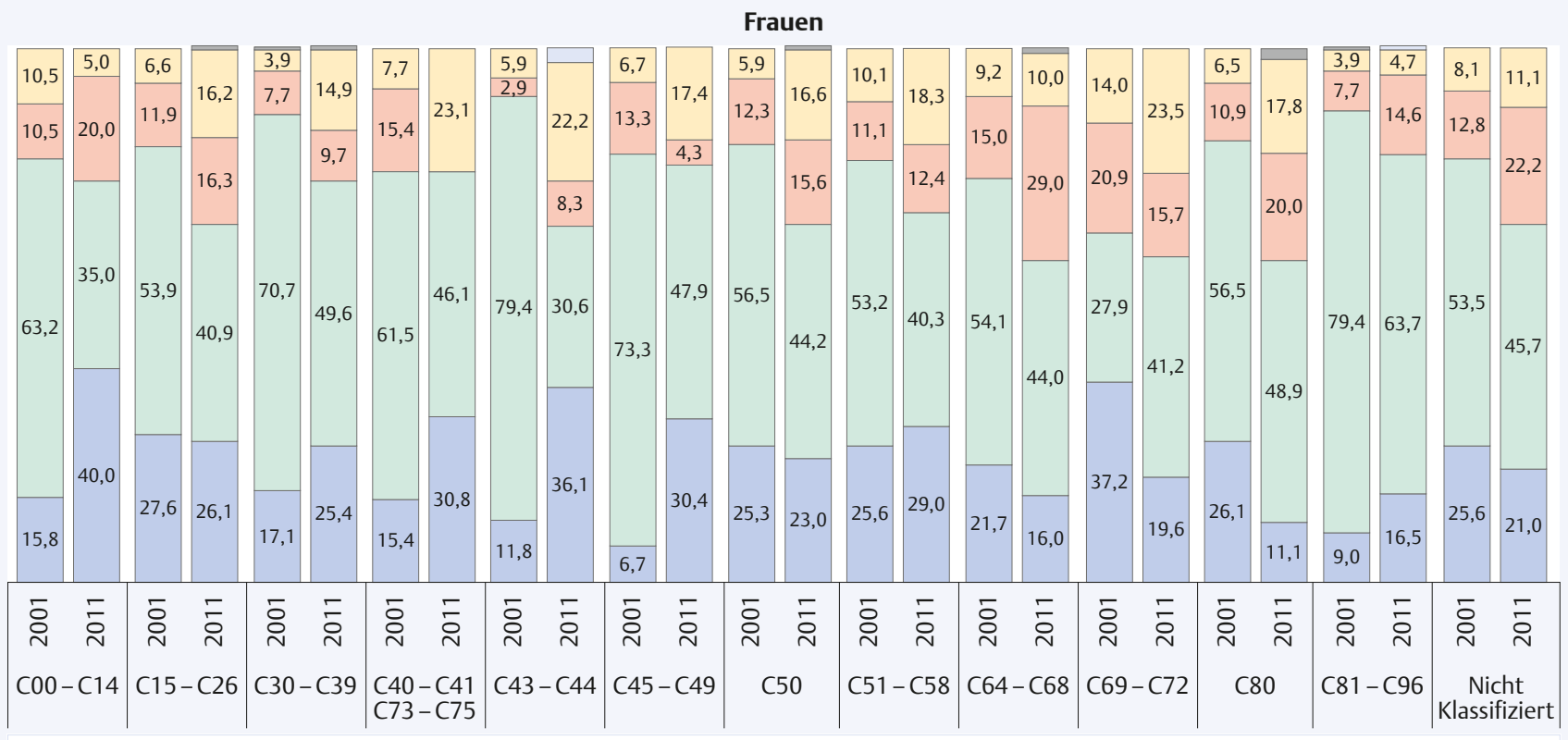

$\square$ Häusliches Umfeld $\quad \square$ Krankenhaus $\quad \square$ Alten- od. Pflegeheim $\quad \square$ Hospiz $\quad \square$ Sonstiger Ort $\quad \square$ Keine Angabe

Männer

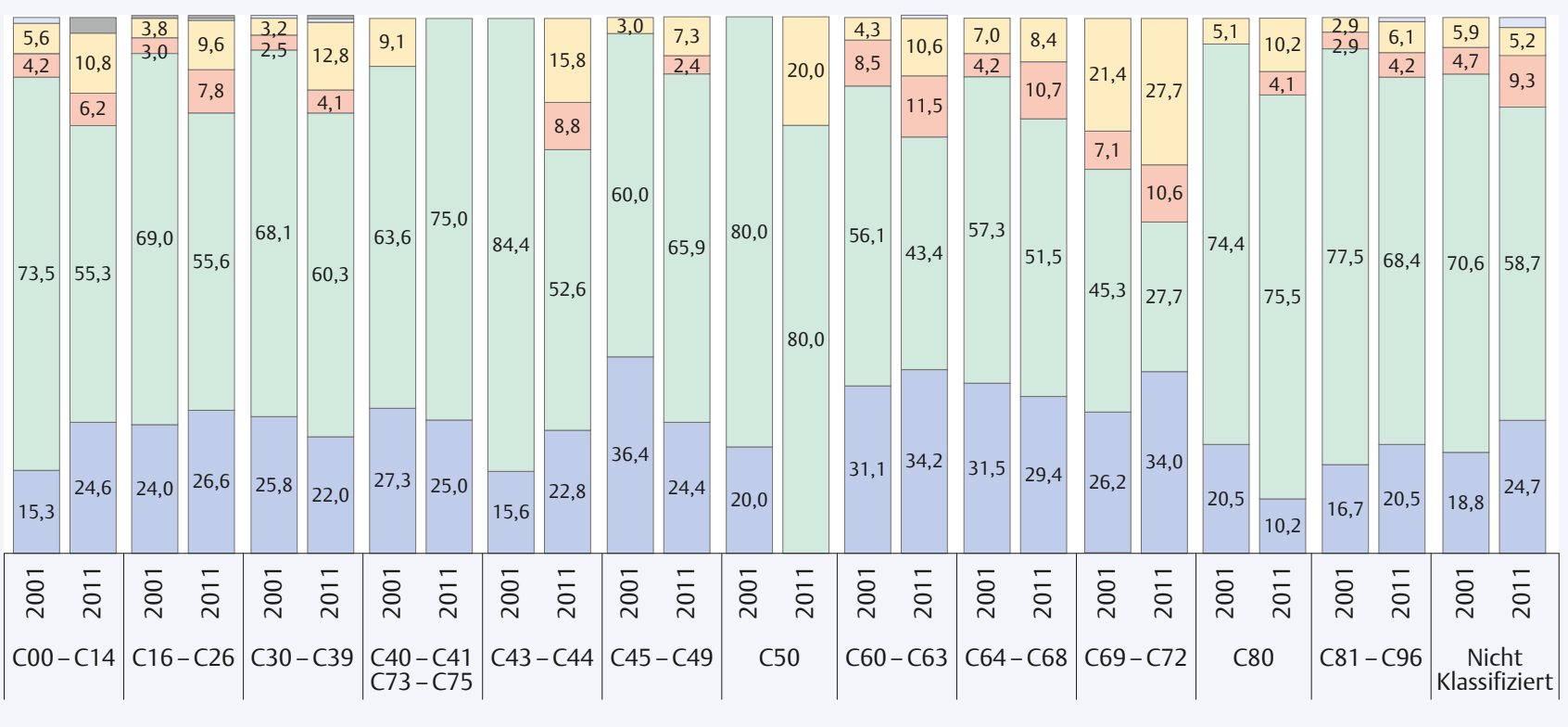

$\square$ Häusliches Umfeld $\square$ Krankenhaus $\quad \square$ Alten- od. Pflegeheim $\quad \square$ Hospiz $\quad \square$ Sonstiger Ort $\quad \square$ Keine Angabe

C00-C14: Lippe, Mundhöhle, Pharynx; C15-C26: Verdauungsorgane; C30-C39: Atmungsorgane und sonstige intrathorakale Organe; C40-C41: Knochen und Gelenkknorpel; C43-C44: Haut; C45-C49: mesotheliales Gewebe und Weichteilgewebe; C50: Brustdrüse;

C51-56: weibliche Genitale; C60-C63: männliche Genitale; C64-C68: Harnorgane; C69-C72: Auge, Gehirn, sonst. Teile des ZNS; C73-C75:

Schilddrüse und sonstige endokrine Drüse; C80: CUP-Syndrom; C81-C96: lymphatisches sowie blutbildendes und verwandtes Gewebe.

Abb. 2 Sterbeort, stratifi-

ziert nach Tumorentität

(C00-C96) und Geschlecht.
(C69-C72) den geringsten Anteil stationärer Sterbefälle auf. Andererseits war bei diesem Patientenkollektiv der Prozentsatz von Sterbefällen im Hospiz sehr hoch (Frauen: 14,0\% bzw. 23,5\%; Männer: 21,4\% bzw. 27,7\%) ( Abb. 2).

\section{Diskussion}

In vorliegender Untersuchung ließ sich ein zeitlicher Trend der Sterbeortverteilung, weg vom
Krankenhaus, hin insbesondere zu Hospizen, Palliativstationen aber auch zu Alten- oder Pflegeheimen beobachten. Trotzdem blieb das Krankenhaus für Tumorpatienten der häufigste Sterbeort. Etwa die Hälfte der Patienten starb stationär. Lediglich jeder vierte Sterbefall ereignete sich im häuslichen Umfeld.

Identifiziert wurden $n=8172$ verstorbene Tumorpatienten, entsprechend einer Häufigkeit von 34\% aller Sterbefälle. Im Vergleich dazu lag 
der Anteil bösartiger Neuerkrankungen an der Gesamtzahl Verstorbener für Deutschland mit 25,1\% im Jahre 2001 (207619 tumorbedingte Sterbefälle auf 828541 Verstorbene) und 26,0\% im Jahr 2011 (221591 Krebstote bei 852328 Verstorbenen) deutlich niedriger [18]. Die beobachteten Differenzen basieren sicherlich zum einen auf Unterschiede im Strukturaufbau beider Populationen, betreffend beispielhaft das Alter oder die Prävalenz von Krankheitsfällen. Andererseits wurden jedoch auch unterschiedliche Methoden zur Identifizierung tumorbedingter Sterbefälle angewendet. Der Ansatz der offiziellen Statistik fußt ausschließlich auf Totenscheinangaben, die im Absatz Todesursache unter dem Grundleiden abgefasst wurden. Des Weiteren nimmt die Statistik lediglich eine Krankheit als Todesursache auf, auch wenn weitere Krankheiten vom Arzt als für den Tod mitbestimmend dokumentiert worden sind. Unser methodischer Ansatz war hingegen so angelegt, dass jegliche verfügbare Arztinformation zur Todesursache für die Feststellung einer Tumorerkrankung herangezogen wurde. Konkret also nicht nur das beschriebene Grundleiden, sondern auch zusätzliche Informationen, dokumentiert z.B. in der ärztlichen Epikrise.

Nationale und internationale Studien bestätigen das überwiegend institutionalisierte Sterben von Tumorpatienten, differieren jedoch in ihren Angaben [6-15]. So führten beispielhaft Cohen et al. [7] in sechs europäischen Ländern (Italien, Belgien, Niederlande, Wales, England, Norwegen) eine Studie zum Sterbeort von Krebserkrankten durch. Hierzu wurden Totenscheine $(n=890750)$ des Jahres 2003 ausgewertet. Die Häufigkeitsverteilung von Todesfällen im Krankenhaus lag in Belgien bei $61,4 \%$, in den Niederlanden bei 31,0\%, in Wales bei $60,1 \%$, in England bei $49,9 \%$ und in Norwegen bei $86,9 \%$ (hier wurden auch Sterbefälle in Pflegeheimen mitgezählt). Für Italien fehlten stationäre Daten. Häusliche Sterbefälle waren in Norwegen mit 12,7\% am niedrigsten und in den Niederlanden mit 45,4\% am höchsten. Für England und Wales lagen zudem Daten für Sterbefälle in Hospizen vor. Hier starben 16,4\% (England) bzw. 7,7\% (Wales) aller Krebspatienten. Für Altenheime lagen die Angaben zwischen 7,6\% (Wales) und 19,2\% (Niederlande).

Verschiedene Einflussfaktoren existieren, die den Sterbeort von Tumorpatienten beeinflussen. Mit einer erhöhten Sterbewahrscheinlichkeit im häuslichen Umfeld sind assoziiert:

- gemeinsames Leben mit einem Angehörigen,

- Möglichkeit der pflegerischen Unterstützung von Familienangehörigen

- verheiratet sein

- Wunsch des Patienten nach einem Sterben in häuslicher Umgebung

- niedriger körperlicher Funktionsstatus

- lange Erkrankungsdauer

- gehobener sozialer Status
- guter Zugang zu Pflegediensten

- hohe Intensität der Pflege

- Wohnen in einer ländlichen Region

Für den Sterbeort Krankenhaus sind bestimmend:

- Vorhandensein einer nicht soliden Tumorerkrankung

- vorherige Einweisung in ein Krankenhaus

- hohes Angebot an Krankenhausbetten

- Wohnen in einer städtischen Region

- Angehörige einer ethnischen Minderheit [19]

In unserer Studie stratifizierten wir die Sterbeortdaten nach Tumorentität und Geschlecht. Personen mit einer bösartigen Tumorerkrankung des Gehirns, sonstiger Teile des ZNS und des Auges (C69-C72) starben überproportional häufig im Hospiz, hingegen seltener im Krankenhaus. Häufig weisen diese Patienten schwer behandelbare Krankheitszeichen wie Hirndruck, epileptische Anfälle, Lähmungen, Koordinationsstörungen sowie kognitive, sprachliche und emotionale Einbußen auf. Dementsprechend ist die pflegerische Versorgung besonders herausfordernd und im häuslichen Umfeld oft nicht mehr zu realisieren. Bei Krebspatienten mit einer bösartigen Neubildung des lymphatischen sowie blutbildenden Gewebes (C81-C96) trat der Sterbefall besonders häufig im Rahmen eines stationären Krankenhausaufenthaltes ein. Oft ist die Prognose bei diesen Patienten schwerer einzuschätzen und sie können auch noch im fortgeschrittenen Krankheitsstadium von onkologischen Therapieangeboten profitieren.

Im Geschlechtervergleich starben Frauen prozentual häufiger im Alten- oder Pflegeheim sowie Hospiz und Männer häufiger Zuhause und im Krankenhaus. Die Lebenserwartung scheint eine entscheidende Rolle zu spielen. Männer weisen aufgrund ihrer niedrigeren Lebenserwartung im Vergleich zu Frauen eine höhere Wahrscheinlichkeit auf, bei bestehender Partnerschaft vom Lebenspartner überlebt und am Lebensende im häuslichen Umfeld von Angehörigen betreut zu werden. Andererseits steigt bei Frauen, bedingt durch das höhere Lebensalter, die Wahrscheinlichkeit, verwitwet oder alleinlebend zu sein. Oft ist bei zunehmender körperlicher Gebrechlichkeit eine häusliche Selbstversorgung nicht mehr zu realisieren. Deshalb werden vermehrt stationäre Versorgungsangebote wie das Alten- oder Pflegeheim in Anspruch genommen.

Unsere Studie umfasste einen Beobachtungszeitraum von 10 Jahren. Im Jahre 2001 existierten in den Städten Bochum und Münster zwei Hospize. Ambulante und stationäre palliativmedizinische Versorgungsstrukturen waren hier erst im Aufbau begriffen. Zehn Jahre später stellte sich das Versorgungsangebot durch Gründung neuer Hospize, Palliativstationen und ambulanter Palliativnetze deutlich verbessert dar. Das Angebot vorge- 


\section{Danksagung}

Die Datenerhebung unterstützten tatkräftig Laila Boutakmant, Hartmut Hofmeister, Marievonne Hofmeister, Viola Willeke. Zudem gilt der Dank den Leitern der Gesundheitsämter Bochum, Borken, Coesfeld und Münster.

\section{Interessenkonflikt}

KB gibt an, Erstattungen für Kongressgebühren sowie Reise- und Übernachtungskosten von Mundipharmaerhalten zu haben. BD, HV und $\mathrm{CB}$ geben an, dass kein Interessenkonflikt besteht. haltener Hospizbetten war von 2,2 auf 4,0 pro 100000 Einwohner angestiegen, sechs neue Palliativstationen wurden eröffnet und in den Städten Bochum und Münster sowie den Landkreisen Borken und Coesfeld waren ab 2006 gegründete Palliativnetze fest in der ambulanten Palliativversorgung etabliert.

Unsere Ergebnisse verweisen auf den Bedarf und die Sinnhaftigkeit der aufgebauten Versorgungsstrukturen. So erhöhte sich u.a. der Anteil von Tumorpatienten, die im Hospiz starben, um mehr als das Doppelte. Andererseits darf das Problem einer angebotsinduzierten Nachfrage hier nicht gänzlich außer Acht gelassen werden. Gleichwohl muss festgehalten werden, dass im klinischen Alltag die Weiterverlegung in ein Hospiz aufgrund zu gering vorgehaltener Bettenzahlen oftmals ein Problem und Nadelöhr darstellt. Krankenhausentlassungen werden hierdurch verzögert und stationäre Liegezeiten verlängert. Dieses Strukturproblem fällt in Abhängigkeit von der Dichte an Hospizbetten auf Bundeslandebene sehr unterschiedlich aus. In diesem Kontext gilt NRW eher als eine Landesregion mit höherem Anteil an stationären Palliativ- und Hospizeinrichtungen. Unsere Daten verweisen auf weitere Problemfelder. Obwohl Untersuchungen zeigen, dass bei der überwiegenden Mehrheit von Tumorpatienten der Wunsch besteht, im häuslichen Umfeld zu sterben, legen die Studiendaten offen, dass dies tatsächlich nur bei jedem vierten Krebspatient der Fall war. Der Anteil häuslicher Sterbefälle blieb trotz des Ausbaus ambulanter palliativmedizinischer Versorgungsstrukturen über 10 Jahre fast konstant, nahelegend, dass dieser Ausbau einer fortwährenden Stärkung bedarf. Sterbefälle von Tumorpatienten im Alten- oder Pflegeheim nahmen kontinuierlich zu.

Stärken und Limitationen | Diese Studie basiert auf dem bislang größten Datensatz ( $n=24009)$, der in Deutschland zum Thema Sterbeort ausgewertet wurde. Zeitliche Trendanalysen wurden durchgeführt. Die Aussagekraft der Untersuchung ist eingeschränkt, da Tumorpatienten anhand von Angaben über die Todesursache aus Totenscheinen identifiziert wurden. Es ist bekannt, dass die Qualität dieser ärztlichen Angaben sehr starken Schwankungen unterlegen ist [20, 21]. Um die Datenqualität maximal hoch zu halten, wurden sämtlich verfügbare Angaben zur Todesursache, einschließlich ärztlicher Epikrise, ausgewertet. Unsere Studie beschränkt sich auf ausgewählte Regionen in Westfalen-Lippe und stellt somit keine Repräsentativität für die Gesamtbevölkerung Deutschlands dar.

Perspektive I Schwerkranke und sterbende Menschen benötigen in ihrer letzten Lebensphase die bestmögliche menschliche Zuwendung, Versorgung, Pflege und Betreuung. Aufgrund des demographischen Wandels wird sich diese Aufgabe in
Zukunft noch aggravieren [13]. Vor diesem Hintergrund scheint das Ziel der politischen Entscheidungsträger, durch Stärkung der Hospiz- und Palliativversorgung in ganz Deutschland ein flächendeckendes Angebot zu verwirklichen, damit alle Menschen an den Orten, an denen sie ihre letzte Lebensphase verbringen, auch im Sterben gut versorgt und begleitet sind, nur gerechtfertigt.

\section{Konsequenz für Klinik und Praxis}

- Tumorpatienten sterben überwiegend in Institutionen.

- Mehr als 50\% sterben im Krankenhaus, knapp ein Viertel Zuhause und annähernd jeder Zehnte im Hospiz oder Alten- bzw. Pflegeheim.

- Im zeitlichen Trend kann eine Verlagerung der Sterbeortes weg aus dem Krankenhaus, hin zu Hospizen, Palliativstationen sowie Alten- oder Pflegeheimen beobachtet werden.

- Patienten mit einer bösartigen Neubildung des ZNS (C69-C72) sterben überproportional häufig im Hospiz, Patienten mit einer bösartiger Neubildung des lymphatischen und hämatopoetischen Gewebes (C81c96) besonders häufig im Krankenhaus

\section{Literatur}

1 Statistisches Bundesamt: Zahlen und Fakten, Gesellschaft und Staat, Gesundheit, Todesursachen.https://www.destatis.de/DE/PresseService/ Presse/Pressemitteilungen/2015/12/

PD15_465_232.html. (Letzter Zugriff: 4. 01. 2016

2 Billingham M], Billingham S]. Congruence between preferred and actual place of death according to the presence of malignant or non-malignant disease: a systematic review and meta-analysis. BM] Supportive \& Palliative Care 2013; 3: 144-154

3 Escobar Pinzon LC, Claus M, Zepf KI et al. Preference for place of death in Germany. J Palliat Med 2011; 14: 1097-1103

4 Gomes B, Higginson IJ, Calanzani N et al. PRISMA. Preferences for place of death if faced with advanced cancer: a population survey in England, Flanders, Germany, Italy, the Netherlands, Portugal and Spain. Ann Oncol 2012; 23: 2006-2015

5 Gomes B, Calanzani N, Gysels M et al. Heterogeneity and changes in preferences for dying at home: a systematic review. BMC Palliat Care 2013; 15, 12: 7

6 Cohen J, Bilsen J, Addington-Hall J. Populationbased study of dying in hospital in six European countries. Palliat Med 2008; 22: 702-710

7 Cohen J, Houttekier D, Onwuteako-Philipsen B et al. Which patients with cancer die at home? A study of 6 European countries using death certificate data. J Clin Oncol 2010; 28: 2267-2273

8 Gomes B, Calanzani N, Higginson IJ. Reversal of the British trends in place of death: Time series analysis 2004-2010. Palliat Med 2011; 26: 102-107

9 Gao W, Ho YK, Verne J et al. Changing Patterns in Place of Cancer Death in England: A PopulationBased Study. PLoS Med 2013; 10: e1001410

10 Broad JB, Gott M, Kim H et al. Where do people die? An international comparison of the percentage of deaths occurring in hospital and residential aged care settings in 45 populations, using published and available statistics. Int J Public Health 2013; 58 : 257-267 
11 Ochsmann R, Slangen K, Feith G et al. Sterbeorte in Rheinland-Pfalz. Zur Demographie des Todes. Interdisziplinärer Arbeitskreis Thanatologie. Beiträge zur Thanatologie. Mainz 1997. Johannes Gutenberg Universität, Heft 8

12 Papke J, Koch R. Places of Death from Cancer in a Rural Location. Onkologie 2007; 30: 105-108

13 Simon ST, Gomes B, Koeskeroglu P et al. Population, mortality and place of death in Germany (1950-2050) - implications for end-of-life care in the future. Public Health 2012; 126: 937-946

14 Thönnes M. Sterbeorte in Deutschland: eine soziologische Studie. Peter Lang Verlag 2013, Frankfurt

15 Dasch B, Blum K, Bausewein C. Sterbeorte: Veränderung im Verlauf eines Jahrzehnts: eine populationsbasierte Studie anhand von Totenscheinen der Jahre 2001 und 2011. Dtsch Arztbl Int 2015; 112: 496-504

16 Entwurf eines Gesetzes zur Verbesserung der Hospiz- und Palliativversorgung in Deutschland (Hospiz- und Palliativgesetz - HPG). http://www. bmg.bund.de/fileadmin/dateien/Downloads/ Gesetze_und_Verordnungen/GuV/H/150612 HPG_GE.pdf. (Letzter Zugriff: 10. 01. 2016)
17 Landesbetrieb Information und Technik NordrheinWestfalen (IT.NRW): https://webshop.it.nrw. de $/$ ssearch.php?kategorie $=1200 \&$ prefix=A10. (Letzter Zugriff: 4. 1. 2016)

18 Gesundheitsberichterstattung des Bundes. Sterblichkeit nach ausgewählten Todesursachen. http://www.gbe-bund.de/gbe10/ergebnisse.prc _ tab? fid $=825 \&$ sprache $=$ D\&fund typ $=$ TXT\&verwandte $=1 \&$ p_Ifd_nr $=45 \&$ p_ uid=gast $\&$ p_aid $=20712887 \&$ hlp_nr $=2 \&$ p sprachkz=D\&p_news=\&p_window $=N \&$ p_janein $=J$. (Letzter Zugriff: 4. 1. 2016)

19 Gomes B, Higginson IJ. Factors influencing death at home in terminally ill patients with cancer: systematic review. BMJ 2006. DOI: 10. 1136/bmj. 38740.614954. 55

20 Cohen J, Bilsen J, Miccinesi G et al. Using death certificate data to study place of death in 9 European countries: opportunities and weaknesses. BMC Public Health 2007; 8, 7: 283

21 Schelhase T, Weber S. Mortality statistics in Germany. Problems and perspectives. Bundesgesundheitsblatt Gesundheitsforschung Gesundheitsschutz. 2007; 50: 969-976
DOI 10.1055/s-0042-111296

Dtsch Med Wochenschr 2016; 141: e158-e165 (c) Georg Thieme Verlag KG . Stuttgart · New York . ISSN 0012-0472 